\title{
Hormonal Changes in Intracranial Hemorrhage
}

\author{
Rajendra Shrestha, ${ }^{1,4}$ Reeka Pradhan, ${ }^{2}$ Mahesh Man Pradhan, ${ }^{3}$ Chao You ${ }^{4}$ \\ 'Department of Neurosurgery, NAMS, Bir Hospital, Nepal, ${ }^{2}$ Paropkar Maternity and Women's Hospital, Thapathali, Nepal, \\ ${ }^{3}$ Department of Surgery, Sumeru Hospital, Nepal, ${ }^{4}$ Department of Neurosurgery, West China Hospital, Sichuan University, \\ China.
}

\section{ABSTRACT}

Introduction: The purpose of this study was to investigate the changes of serum ACTH and Thyroid hormone level in the acute phase of ICH.

Methods: Sixty patients with spontaneous ICH were assessed regarding hormonal changes by measuring ACTH and Thyroid hormone level. The relationships of hormone concentrations to clinical and radiological parameters were evaluated at hospital admission (t0), 5 days (t1) and 10 days (t2). The results were statistically analyzed.

Results: ACTH, TSH, Thyroxine, and free Thyroxine were not significantly different among the three time periods $(\mathrm{P}>0.05)$, while Triiodothyronine (T3) and free Triiodothyronine (FT3) were significantly different $(\mathrm{P}<0.05)$. T3 and FT3 were not significantly different among patients with hemorrhage of different locations $(\mathrm{P}>0.05)$. There were significant negative correlations between $\mathrm{T} 3$ and FT3 with volume of $\mathrm{ICH}(\mathrm{r}=-0.63$ and $\mathrm{r}=-0.25)$ and there were positive correlations between T3 and FT3 with GCS ( $r=0.63$ and $r=0.37)$ respectively on admission day $(p<0.05)$.

Conclusions: Hormonal secretion patterns is associated with the severity of ICH. This is an important index to evaluate the disease severity and prognosis.

Keywords: ACTH; ICH; lymphocyte ICH; Thyroid hormone.

\section{INTRODUCTION}

Intracerebral hemorrhage $(\mathrm{ICH})$ is a major clinical issue, causing up to $15-20 \%$ of all strokes and subsequent death or severe disability in $50-70 \%$ of patients even in the presence of recent therapeutic progress. It estimated to disturb over one million people worldwide each year. ${ }^{1}$ The coagulation cascade, heme catabolism products and inflammation play a part in $\mathrm{ICH}$ induced injury. A lot of changes occurs in the brain including hematoma formation, brain edema, inflammation and microglia activation. ${ }^{2}$ That leads to fluctuation in hormonal and immunological parameters temporarily or permanently.

We have analyzed the hormonal profiles status of $\mathrm{ICH}$ patients due to the important interactions between the central nervous system and the endocrine system, particularly in stressful situations. Because of the complex pattern of $\mathrm{ICH}$ induced cerebral phenomena proper prevention and treatment of the consequences of this pathology will probably need a multimodal approach comprising measures aimed at $\mathrm{ICH}$ associated immune, hormonal and inflammatory processes. ${ }^{3,4}$

Correspondence: Dr. Rajendra Shrestha, Department of Neurosurgery, NAMS, Bir Hospital, National Trauma Centre, Nepal. Email: rajendra39@yahoo.com, Phone: +977-9851063830. 


\section{METHODS}

Sixty patients of both sexes with spontaneous $\mathrm{ICH}$ were admitted consecutively and qualified for the study from May 2013 to Oct 2013 in the Cerebrovascular Hypertensive Unit of Neurosurgery Department of the "West China Hospital, Sichuan University". Eighteen females (age: $35-70$ years; mean \pm SD: $53 \pm 10.1$ years) and forty two males (age: $28-83$ years; mean \pm SD: 58.8 \pm 14 .5years) with $\mathrm{CT}$ verified acute $\mathrm{ICH}$ patients were included in the study. The difference between the age of females and males were not statistically significant $>0.05)$. Eight patients were classified in mild (13-15), forty seven in moderate group (9-12) and only five in severe grade ( 8 or lower) of the Glasgow coma scale (Table 1). This study was approved by the Committee of Ethics in Research of the Neurosurgery Department, West China Hospital, Sichuan University. All patients or their next of kin were given written informed consent. The diagnosis of spontaneous $\mathrm{ICH}$ was established by physical findings and brain Computerized Tomography (CT) or Magnetic Resonance Imaging (MRI). The $\mathrm{ICH}$ volume was measured from the first $\mathrm{CT}$ or MRI scan using $a \times b \times c \times 0.5$ method. For irregular shapes, the different formula $a \times b \times c \times 0.33$ was used.$^{5,6}$ Patients or their legal representatives signed the informed consent for participation in the study. Basic clinical parameters, history of hypertension and previous antihypertensive therapy were included in the analysis. All the patients were followed until discharged from hospital. Before discharge they were also evaluated by the prognostic Glasgow scale.

The inclusion criteria included those $\mathrm{ICH}$ patients who were older than 18 years and non-pregnant, with no immune or endocrine disorders and not suffering from any active infectious disease or a non-aneurysmal ischemic disease. They should not have received any prior chronic glucocorticoid treatment or any hormonal contraceptives in the last 3 months. They should not have received any blood or massive electrolyte infusions during the hospital stay. They should not be malnourished and should not have any associated systemic diseases, such as renal, heart, respiratory failure and Diabetes Mellitus.

The study excluded Subarachnoid hemorrhage cases, patients classified in severe GCS score who refused operation, patients previously using drugs like replacement hormonal therapy of any type, having hemorrhages secondary to trauma, hemorrhages due to malformations, coagulopathy, and tumors, hematological disorders and cases of infection within the first 72 hours.
Venous blood samples were obtained at 3 times: during the first 24 hours of $\mathrm{ICH}$ that is at hospital admission ( $\mathrm{t} 0$ ), 5 days ( 1 ), and 10 days (t2). Blood samples were collected between 8 and 9 AM, within 24 hours from ictus, centrifuged at $3000 \mathrm{rpm}$, for 10 minutes, and the serum stored at $-20{ }^{\circ} \mathrm{C}$ until assayed. Adrenocorticotropic hormone (ACTH), Thyroid stimulating hormone (TSH), T3, FT3, T4 and FT4 were measured by Electrochemiluminescence immunoassay (ECLIA) in serum (Modular E170C, Roche Diagnostics $\mathrm{GmbH}$, Germany).

Data were analyzed by the analysis of the variance (ANOVA) and paired simple T-test for comparisons. Bivariate analysis was performed to investigate the correlation to each other. The level of significance was fixed at $p<0.05$. The results were presented as mean \pm standard deviation $(M \pm S D)$ with help of the Statistical Package for Social Sciences (SPSS) for Windows, version 21.0 (Chicago, IL).

\section{RESULTS}

Twenty one patients managed conservatively, whereas thirty nine patients were symptomatic presenting with acute focal neurological deficits with mass effect on admission. GCS grade, $\mathrm{ICH}$ score and duration of Intensive Care Unit stay were significantly higher in operative and infective patients. Outcome of operative $\mathrm{ICH}$ patients were significantly impaired compared to the conservative group. Five patients died due to pneumonia followed by multi-organ failure and acute respiratory distress syndrome with fatal pulmonary insufficiency at days $17,20,21,25$, and 35 after $\mathrm{ICH}$ respectively.

\begin{tabular}{|ll|}
\hline Table 1. Glasgow come scale of admitted patients. \\
\hline GCS & $\mathrm{n}(\%)$ \\
Mild & $8(13.3)$ \\
Moderate & $47(78.3)$ \\
Severe & $5(8.3)$ \\
Total & $60(100)$ \\
\hline
\end{tabular}

ACTH, TSH, Thyroxine (T4), serum free Thyroxine (FT4) had no significant changes at hospital admission day, 5 days, 10 days. That also indicated that ACTH, TSH, T4, FT4 level were not statistically significant $(P>0.05)$ in both operative and conservative cases. Different sites of $\mathrm{ICH}$ with serum hormonal level at hospital admission (t0), 5 days ( $\mathrm{t} 1)$, and 10 days (t2) were not statistically significant $(P>0.05)$. 
Table 2. The relation of ACTH, TSH, T3, T4, FT3 and FT4 in both operative and conservatively patients by OneWay ANOVA statistic.

\begin{tabular}{|c|c|c|c|c|}
\hline Hormone Types & “0”day & “5” days & "10" days & $\mathrm{P}$ value \\
\hline ACTH & $22.81 \pm 18.53$ & $26.24 \pm 19.51$ & $26.22 \pm 13.92$ & 0.570 \\
\hline TSH & $1.33 \pm 1.82$ & $1.51 \pm 1.86$ & $1.87 \pm 1.82$ & 0.375 \\
\hline T3 & $1.35 \pm 0.37$ & $1.01 \pm 0.32$ & $0.72 \pm 0.43$ & $<0.001$ \\
\hline FT3 & $3.61 \pm 0.95$ & $2.64 \pm 0.79$ & $2.10 \pm 1.02$ & $<0.001$ \\
\hline T4 & $98.24 \pm 22.92$ & $99.80 \pm 22.26$ & $94.19 \pm 20.48$ & 0.158 \\
\hline FT4 & $16.10 \pm 3.56$ & $15.22 \pm 3.26$ & $15.23 \pm 3.30$ & 0.190 \\
\hline
\end{tabular}

Serum Triiodothyronine (T3) and free Triiodothyronine (FT3) were gradually at 5 days (t1) and significantly decreased at 10 days than hospital admission (tO) in both operatively and conservatively managed cases (Figure 1,2). T3, FT3 of both operative and conservative cases together at hospital admission day 5 days, 10 days were statistically significant $(P<0.05)$ (Table 2). However, T3 and FT3 level were not significant ( $P$ $>0.05$ ) in between operative and conservative cases. In case of conservatively management cases, T3, FT3 levels were significantly decreased 5 days (t 1$)$, then gradually raised at 10 days (during discharge time).

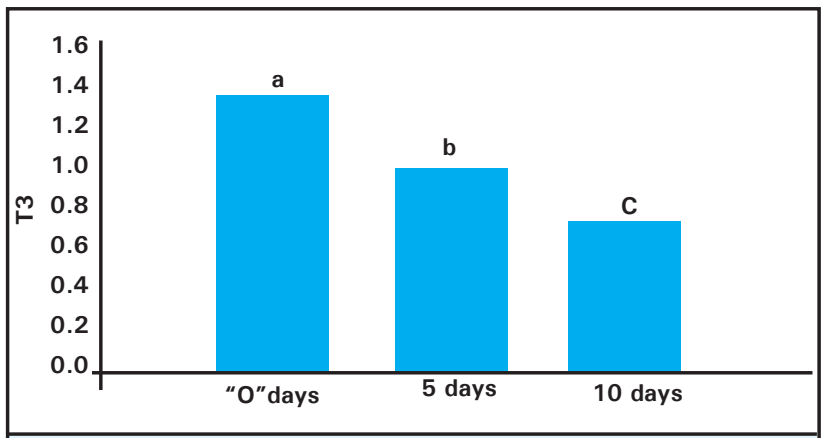

Figure 1. Showing different pattern of T3 level in at hospital admission ( 0 days), 5 days and 10 days. Do not share a same letter are significantly different $(P<0.05)$.

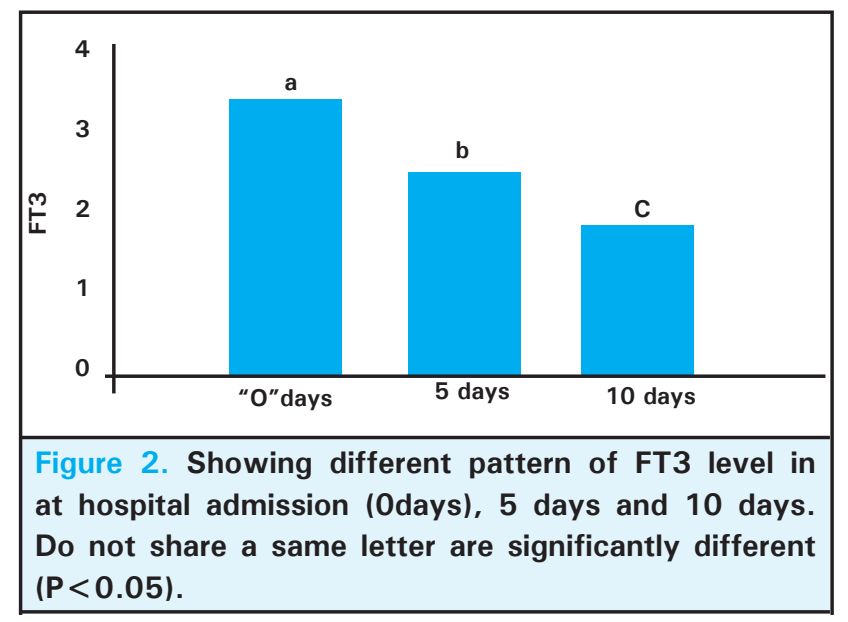

There were significant negative correlations between T3 and FT3 with volume of ICH ( $r=-0.63$ and $r=-0.25)$ and there were positive correlations between T3 and FT3 with GCS ( $r=0.63$ and $r=0.37)$ respectively on admission day $(p<0.05)$.

\section{DISCUSSION}

Intracerebral hemorrhage $(\mathrm{ICH})$ is a common type of fatal stroke, accounting for about $15-20 \%$ of all strokes. Hemorrhagic strokes are related with high mortality and morbidity, and increasing evidence endorses that innate immune responses and inflammatory injury play a critical role in $\mathrm{ICH}$ induced neurological deficits. ${ }^{2}$ During acute $\mathrm{ICH}$, immediate accumulation of blood within brain parenchyma leads to interruption of normal anatomy and raised local pressure. Depending on the hematoma growth, the primary injury arises within minutes to hours from the onset of bleeding and is primarily the result of mechanical damage associated with the mass consequence. Secondary damage is for the utmost part due to the presence of intra-parenchymal blood may be dependent upon the initial hematoma location, ventricular volume, onset of hemorrhage and age of patients. It may occur through many parallel pathological pathways including: (1) cytotoxicity of blood, (2) stimulate toxicity, (3) hyper metabolism, (4) spreading depression, and (5) oxidative stress and inflammation. ${ }^{7,8,9}$ Ultimately, these pathogenesis leads to irreversible interruption of the constituents of the neurovascular unit, gray and white matter, and is followed by blood brain barrier interference and deadly brain edema with huge brain cell death. While inflammatory mediators produced locally in reaction to injury or brain death have the ability to enhance damage triggered by $\mathrm{ICH}$ (secondary injury). The involvement of inflammatory cells e.g. microglia or macrophages is important to remove or cleanup of cellular debris from hematoma and the source of continuing inflammation. The well timed removal of damaged tissue is crucial for reducing the length of deleterious pathological process and thereby allowing for faster and more efficient 
improvement. ${ }^{10}, 11,12,13$ Although, there is much study on the condition following $\mathrm{ICH}$, the initial cellular events activate innate immune and inflammatory responses are still complex. Therefore, an approach for suppressing the inflammatory responses is seriously needed.

In our first study, we had investigated Serum ACTH, Thyroid hormone (TSH, T3, T4, FT3 and FT4) levels of the sixty cases of intracranial hemorrhage patients. We identified that ACTH, TSH, T4 and FT4 levels were within the normal range in group of preoperative, postoperative patients and also conservatively managed cases. But, T3 and FT3 levels preoperative and postoperative were lower than normal and emergence as "low T3 syndrome". T3 and FT3 reduced the more clearly in severe the disease; preoperative T3, FT3 measured value is low to normal, after $5 \mathrm{~d}$ lower than the preoperative, $10 \mathrm{~d}$ gradually more decreased in post-operative patients (Figure 1,2). All these changes due to $\mathrm{ICH}$ lead to surgery which related to hypothalamic pituitary thyroid axis dysfunction.T3, FT3 level of preoperative and postoperative patients were altered at hospital admission ( $\mathrm{t} 0$ ), 5 days ( $\mathrm{t} 1$ ), and 10 days ( $\mathrm{t} 2$ ). These are possible mechanism to change serum hormonal level in acute ICH: (1) bleeding leads to increased intracranial pressure, cerebral edema and hypothalamic injury (2) bleeding after the stress response, the pituitary secretion increased (3) brain injury can be triggered by hypothalamic pituitary thyroid axis dysfunction. Thyroid cycle secretion was repressed by acute injury leads to T3 hormone reduction. (4) Craniotomy and mobilization of the brain tissue, postoperative cerebral edema and other factors directly damage the hypothalamus and (5) Release bloody cerebrospinal fluid which help alleviate cerebral edema and increased intracranial pressure. ${ }^{14,15}$

During surgical procedure, remove the hematoma, the release of hematoma induce mass effect and clean up the blood product on the brain to reduce the stimulation of brain tissue and blood vessels, subside cerebral edema and other factors gradually lower the damage to the hypothalamus, the hypothalamic pituitary thyroid axis function gradually stabilized. That groups of patients who had GCS were mild during operation and medically treated patients, serum hormone levels improved comparatively at 10 days from 5 days for the following reasons: (1) this group of patients is in a stable condition after surgery (usually about one week) and transferred to rehabilitation hospital, (2) Before surgery, patients had acute $\mathrm{ICH}$ hormonal level, (3) peak of brain edema usually appear in postoperative 3-4 days, (4) After 4-5 days, peak of brain edema disappears, then patient's condition is stabilized. ${ }^{16}$ In contrast to the above, preoperative and postoperative hormone levels found that only T3, FT3 are statistically significant, we also revealed that T3, FT3 levels cause significant changes in clinical observation, expressed as "low T3 syndrome".T3, FT3 levels were altered with the patient's condition i.e. lower the levels of T3, FT3, the more severe the disease. After stable condition, T3, FT3 levels gradually rise into normal hormone levels, consistent with the statistical results.

These days, a large number of studies have established that multiple trauma, respiratory failure, pulmonary heart disease and cirrhosis cause significant reduction in Thyroid hormones in patients with acute brain injury in T3. Most of the authors understood that critically ill patients should be stimulated by the body's endocrine system during a compensatory protection from the disease. The lower the T3 level was found in more severe the diseases. This indicated that T3 levels are associated with critically ill patient's condition. ${ }^{14,16,17}$ In our opinion, "low T3 syndrome" patients are required to correct hormone supplements externally. It can be hold when they come to normal. Elovic et al recommended that hormone replacement therapy for hypothyroid patients, hypothalamic damage induce alternation of Thyroid function caused by primary or secondary trauma. Vespa et al reported that hormone replacement therapy for acute or chronic phase of hypothalamic pituitary adrenal axis dysfunction treatment is ineffective or even harmful. In our study group, patients with $\mathrm{ICH}$ before and after surgery were not supplemented hormone, T3 and FT3 levels returned to normal when the condition gradually improved, that is "low T3 syndrome" is gradually corrected. ${ }^{18,19} \mathrm{~A}$ major limitation of our study is the limited patient number. Although it is emerging that brain hormonal interactions after $\mathrm{ICH}$ have severe clinical suggestions regarding the result, a better understanding of the hormonal response of this complex pathophysiological condition is essential and may lead to effective beneficial strategies, eradicating a chief factor of complications in $\mathrm{ICH}$ patients. This is the first clinical study to find out the relation between $\mathrm{ICH}$ and T3 and FT3 in English and Chinese literatures.

\section{CONCLUSIONS}

T3 and FT3 were significant in operatively managed ICH cases. These parameters change and indicate prognosis of intracranial hemorrhage patients due to $\mathrm{ICH}$ induced hypothalamic pituitary thyroid axis dysfunction. This kind of information is an important prognostic indicator to guide the early detection of patient's clinical condition changes, to make adjustments early in the treatment plan.

Conflict of Interest: None. 


\section{REFERENCES}

1. Kase CS, Caplan LR. Intracerebral Hemorrhage. Newton, MA: Butterworth-Heinemann; 1994.

2. Qureshi AI, Tuhrim S, Broderick JP, Batjer HH, Hondo H, Hanley DF. Spontaneous Intracerebral Hemorrhage. N Engl J Med. 2001;344:1450-60. [PubMed]

3. Reincke M, Allolio B, Wurth G, Winkelmann W. The hypothalamic-pituitary-adrenal axis in critically ill patients: response to $\mathrm{CRH}$ and Dexamethasone. J Clin Endocrinol Metab. 1993;77:151-6. [PubMed]

4. Siesjö BK \& Siesjö P. Mechanisms of secondary brain injury. Eur J Anaesthesiol. 1996;13:24768. [PubMed]

5. Kothari RU, Brott T, Broderick JP, Barsan WG, Sauerbeck LR, Zuccarello M, Khoury J. The ABCs of measuring intracerebral hemorrhage volumes. Stroke. 1996;27:1304-5. [Full Text]

6. Huttner HB, Steiner T, Hartmann M, Ko“hrmann M, Juettler E, Mueller S, Wikner J, Meyding-Lamade U, Schramm P, Schwab S, Schellinger PD. Comparison of ABC/2 estimation technique to computer-assisted planimetric analysis in Warfarin-related intracerebral parenchymal hemorrhage. Stroke. 2006;37:404-8. [Full Text]

7. Xi G, Keep RF, Hoff JT. Mechanisms of brain injury after intracerebral haemorrhage. Lancet Neurol. 2006;5:53-63. [PubMed]

8. Aronowski J,Hall CE. New horizons for primary intracerebral hemorrhage treatment: Experience from preclinical studies. Neurol Res. 2005;27:268-79. [pubMed]

9. Gong C, Hoff JT, Keep RF. Acute inflammatory reaction following experimental intracerebral hemorrhage in rat. Brain Res. 2000;871:57-65. [PubMed]

10. Wang J, Dore S. Inflammation after intracerebral hemorrhage. J Cereb Blood Flow Metab. 2007;27:894-908. [PubMed]
11. Huang FP, Xi G, Keep RF, Hua Y, Nemoianu A, Hoff JT. Brain edema after experimental intracerebral hemorrhage: Role of hemoglobin degradation products. J Neurosurg. 2002;96:287- 93. [PubMed]

12. Wang X, Mori T, Sumii T, Lo EH. Hemoglobin-induced cytotoxicity in rat cerebral cortical neurons: Caspase activation and oxidative stress. Stroke. 2002;33:1882-88. [PubMed]

13. Zhao X, Sun G, Zhang J, Strong R, Song W, Gonzales N, et al. Hematoma resolution as a target for intracerebral hemorrhage treatment: Role for peroxisome proliferatoractivated receptor gamma in microglia/macrophages. Ann Neurol. 2007;61:352-62. [PubMed]

14. Elvio Scoscia, Stefano Baglioni, Amir E, et al. Low triiodothyronine (T3) state: a predictor of outcome in respiratory failure? Results of a clinical pilot study [J]. Endocrinology.2004;151(5):557-60. [PubMed]

15. Elovic EP, Glenn MB. Anterior pituitary dysfunction after traumatic brain injury II. J Head Trauma Rehabil. 2004;19(2):184-7. [PubMed]

16. Chrousos GP. The hypothalamic-pituitary-adrenal axis and immune-mediated inflammation. $N$ Engl J Med 1995;332:1351-62. [PubMed]

17. Vaughan GM, Becker RA, Allen JP, Goodwin CW, Pruitt BA, Mason AD. Cortisol and corticotropin in burned patients. J Trauma 1982;22:263-72. [uㅏㄹed]

18. Brent GA, Hershman JM, Braunstein GD. Patients with severe nonthyroidal illness and serum thyrotropin concentrations in the hypothyroid range. Am J Med 1986;81: 463-6 [PubMed]

19. Wastofsky L, Burman KD. Alterations in thyroid function in patients with systemic illness: the "euthyroid sick syndrome." Endocr Rev. 1982;3:164-217. [PubMed] 